\title{
CCD ASTEROSEISMOLOGY OF OPEN CLUSTERS
}

\author{
INMACULADA VIDAL, JUAN A. BELMONTE \\ Instituto de Astrofísica de Canarias, c/Via Láctea s/n 38200, La Laguna, \\ Tenerife, Spain.
}

In the last few years Asteroseismology has contributed to the knowledge of stellar interior, in some extend. This contribution has been done with observations, providing the characteristics of the acuostic modes of stellar oscillations; and with theory relating them to inner parameters, locating stars in the JCD diagram (Christesen-Dalsgaard J., 1989). At the beginning only classical pulsating stars had high enough amplitudes able to be detected. But techniques have been improved and now the goal is the detection of oscillations in all kind of stars, specially solar-like ones. Up to now, few cases of possible detections have been reported, the best evidences are: Belmonte et al, (1990) in an FV star, Brown et al (1991) on the subgigant Procyon, and Pottasch et al (1992), in $\alpha$ Cen.

In order to reach precision high enough, CCD photometry has been used in the last few years, as an alternative and more powerful way. This new technique has already been applied by other groups such as $R$. Gilliland and $T$. Brown in the USA (Gilliland $\epsilon t$ al,1991) and S. Frandsen and H. Kjeldsen in Denmark (Kjeldsen and Frandsen, 1992) with promising results. Our group has two data series one of NGC6802 (observed with the $2.5 \mathrm{~m}$ INT at ORM, Canary Islands, Spain), and the other of a $\delta$ Scuti, BQCnc, in Praesepe cluster (observed with the 1.m JKT at the same observatory). W'ith the last one, we expect to compare limits and characteristics between CCDs and photometers. At present we are finishing up with the reductions of NGC6802. In order to obtain the light curves of stars in the field, we have used aperture photometry (only when the crowdness of the field is not very high) and a more general PSF fitting. Some initial results on NGC6802 analysis are presented in this report.

Besides, with a 2.5 meter telescope, observing a 15 th magnitude star, the noise level is dominated by counting noise. The selected stars range of magnitudes is 15 to 17 , and one 13th magnitude star, so photon noise will be the most important component of noise in spectra.

\section{REFERENCES}

Belmonte J.A., Pérez Hernández F. and Roca Cortés T., 1990. Astron. Astr. 231, 383.

Belmonte J.A., Chevreton M., Mangeney A., Praderie F., Saint-Pé O., Puget P., Alvarez M., and Roca-Cortés T., 1991. Astron. Astr. 246, 71-83. 


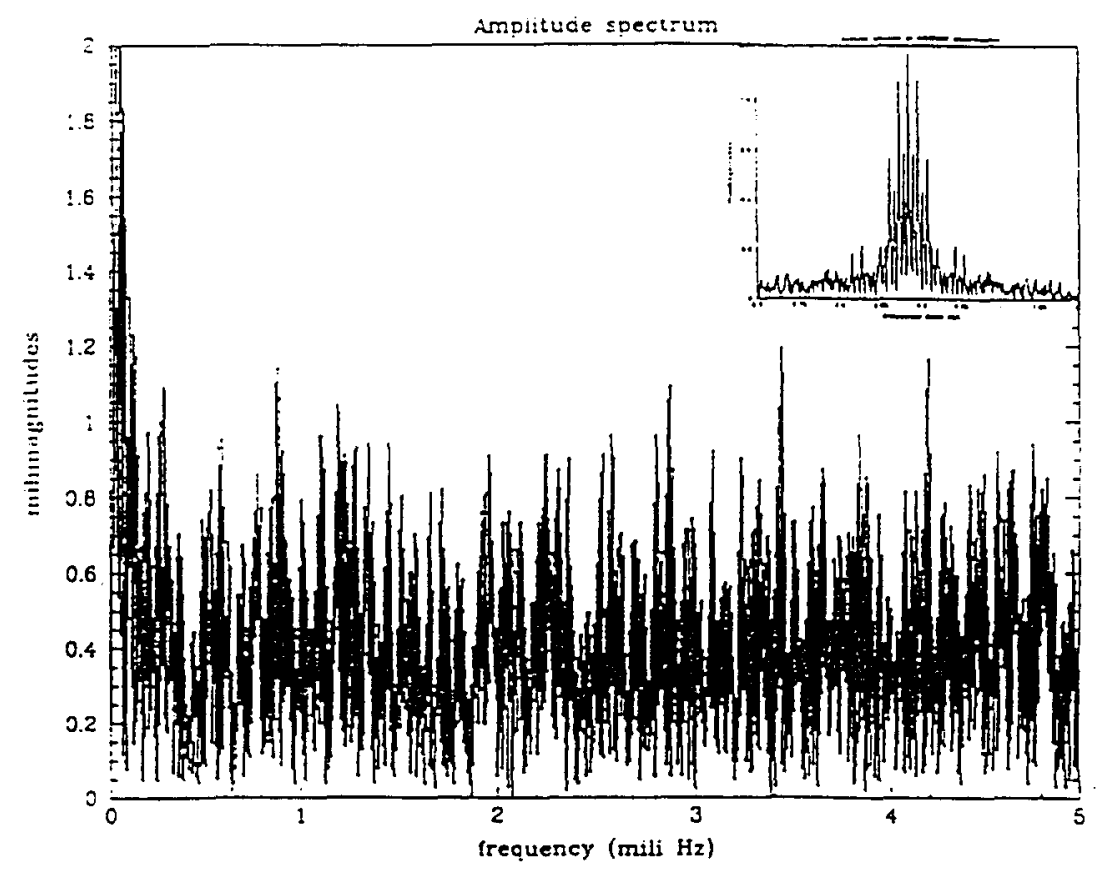

FIGURE I Amplitude spectrum of a 15th magnitude star in NGC6802. Residual series are tipycally 6 hours long, with an exposure time of $50 \mathrm{sec}$. It has been obtained via an iterative sinewave fitting procedure (Belmonte et al, 1991) applied to five series $(9,10,11,12,14$ June 1990). Noise level is about 530 micromagnitudes, althouhg expected peaks for solar-like stars are well below this level, (around $50 \mu \mathrm{mag}$ for the best expectations) still some information could be obtained from this kind of spectra. Spectral window is plotted as well. The gaps in data make it wide, so possible peaks are dificult to detect. 


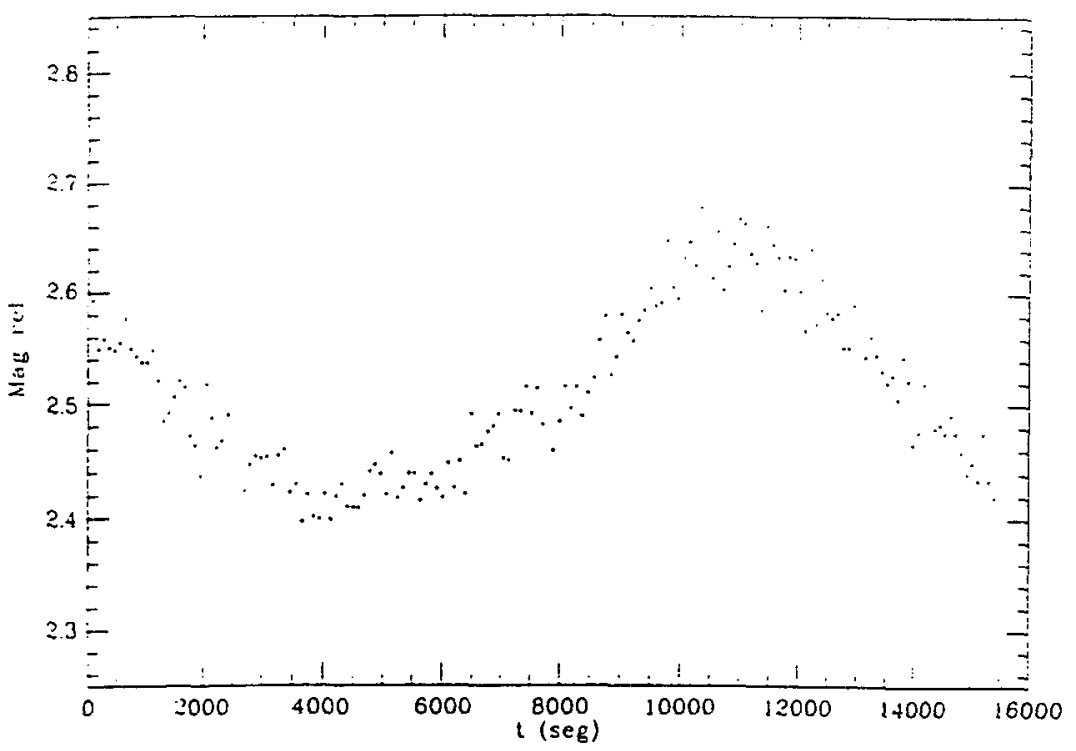

FIGURE II Light curve of a variable star found in NGC6802. From its amplitude and aproximate period we suppose it is a W UMa variable. This curve has been obtained with the computer of Nordic Optical Telescope, at the ORM, using Hans Kjeldsen's reduction programs, called MOMF, which are based on a combine PSF fit and aperture photometry algorithms.

Brown T.W., Gilliland R.L., Noyes R.L. and Ramsey R.W., 1991. Ap. J., 368, 599.

Christensen-Dalsgaard. J., 1989. 'Lecture Notes on Stellar Oscillations' Univ. de Aarhus.

Gilliland R.L., Brown T.W., Duncan D., Suntzeff N.B., Schild R., Jeffrey W.A. and Penprase B., 1991. Astron. J., 101, 541.

Kjeldsen H. and Frandsen S., 1992. P.A.S.P., in press.

Pottasch E.M., Butcher H.R. and van Hoesel F.H.J., 1992, submitted.

Stetson P.B. 198i. P.A.S.P. 99, 191. 